\title{
A Stall-Regulated Wind Turbine Design to Reduce Fatigue
}

\author{
Terence Macquart ${ }^{1}$, Alireza Maheri ${ }^{2}$ \\ 1. Department of Aerospace Engineering, University of Bristol, Bristol, UK \\ 2. School of Engineering, University of Aberdeen, Aberdeen, UK
}

\begin{abstract}
Variable-speed stall-regulated (VS-SR) wind turbines can be designed to produce power as efficiently as variable-speed pitch-controlled (VS-PC) systems. However, amongst the main drawbacks of VSSR systems high transient power and low predictability have been the primary factors in favour of adopting VS-PC system for multi-MW wind turbines. Cyclic and stochastic loads leading to fatigue failure is one of the prime considerations for large wind turbines. In contrast to the current trend of research, which is focused on load alleviation by integrating active flow controllers, this paper highlights the potential benefits of VS-SR wind turbines in reducing fatigue loads. Adopting the NREL 5MW wind turbine as the baseline, blades are redesigned for stall-regulation. It is shown that a well-designed VS-SR wind turbine experiences significantly less fatigue loads compared to VS-PC systems. It also results in low power transients near and above rated wind speed. Taking into account added complexity, mass and maintenance costs of wind turbines utilising active flow controllers and in view of the recent progresses that have been made regarding the aeroelastic stability of stalled blades, VS-SR systems seem to have a role to play in the design of future wind turbines.
\end{abstract}

Keywords: fatigue load alleviation; aerodynamic sensitivity; progressive stall line; variable-speed; stall-regulated wind turbine; WTAC

\section{Introduction}

The incentive in reducing the cost of wind energy has led to a steady growth in wind turbines rotor size over the past decades. However, major technical challenges are to be overcome in order to maintain the current growth rate. In particular, blade failure due to fatigue has become a major design concern [1-3]. Variable-speed pitch-controlled (VS-PC) wind turbines are designed for maximising power generation and keeping the aerodynamic torque at nominal value [4]. Compared to variablespeed stall-regulated (VS-SR) wind turbines, VS-PC have lighter blades and produce less noise. Moreover, VS-PC have a well-understood and predictable aerodynamic under attached flow, high aerodynamic damping and a refine power control. On the other hand, VS-PC wind turbines also have limitations. Wind turbines cyclic loads, arising due to the cyclic motion of the blades in a nonaxisymmetric wind field, are the prime cause of fatigue [5, 6]. The collective pitch control strategy for VS-PC turbines is not designed for relieving fatigue loads. Furthermore, the most commonly employed pitch control strategy (i.e. pitch to feather) maintains the blades in attached flow conditions resulting in high aerodynamic sensitivity causing large alternating fatigue loads. The integration of new active flow controllers (e.g. trailing edge flaps and microtabs) to alleviate fatigue loads increases the complexity, mass and maintenance costs of wind turbines. While the trend in current research focuses on alleviating the loads of VS-PC wind turbines using active flow controllers [7-10], the present paper highlights the potential benefits of employing variable-speed stall-regulated (VS-SR) wind turbines. In particular, this paper seeks at raising interest in fatigue and transient power reduction by taking advantage of the low aerodynamic sensitivity of stalled blades.

Most investigations on VS-SR wind turbines occurred more than a decade ago. During that time the main arguments driving the VS-SR research were the substantial reduction in installation and maintenance costs as well as lighter blades and simpler control systems compared to VS-PC wind turbines [11-14]. Investigations have shown that a few VS-SR control strategies could be used to maximise and limit power at low and high wind speeds [11-15]. However, the generator and converter size of VS-SR turbines had to be increased [16] in order to absorb high power transients occurring 
due to sudden changes in wind speeds (e.g. gust). It is important to note that at that the wind turbines on which the VS-SR control strategies were tested were not especially designed for this type of operating conditions. A recent investigation [17] suggested that specifically designed blades could have better dynamics in stall such as lower aerodynamic sensitivity and higher aeroelastic stability. Recent research has also shown that the blade tip design (e.g. back-twist) plays a critical role in generating aerodynamic damping [17-19]. Although it has long been known that stall-regulated blades have a low aerodynamic sensitivity, the present investigation provides a thorough investigation using this knowledge to reduce fatigue loads and transient dynamics.

The rest of this paper is structured as follows. The design of a large-scale VS-SR wind turbine is proposed in Section 2. The aerodynamic sensitivity and fatigue of both wind turbine designs are compared in Section 3. The variable-speed control performance of the VS-SR wind turbine is investigated in Section 4. The outcomes of this investigation are summarised in Section 5.

\section{Variable-Speed Stall-Regulated Design}

The aeroelastic code WTAC [20] is used for calculating the wind turbines aerodynamic performance. WTAC (Wind Turbine Aeroelastic and Control) was originally developed to calculate the aerodynamic and structural performance of wind turbines equipped with active flow controllers and to ease the development and evaluation of control structures and strategies for actively controlled wind turbine blades. WTAC includes an unsteady BEMT as well as a structural, a control and a wind field module. WTAC predictions are compared with FAST [21] for the NREL 5MW wind turbine [22] in Figure 1.

The original blade design of the 5MW VS-PC wind turbine is not suitable for stall-regulated operation and the chord, twist and aerofoil distributions have to be re-designed. The stall-regulated design is obtained by using a genetic algorithm coupled with WTAC steady state BEMT in order to maximise power and minimise thrust. The final values of the design variables found for the stall-regulated design are detailed in Figure 2 and Table 1. Note that this paper aim is not to find the optimal design, including all aerodynamic and structural design variables, but rather to demonstrate the advantages of VS-SR wind turbines for large scale applications.

In comparison to pitch-controlled systems, three dimensional stall [23] has a non-negligible influence on the power and thrust predictions of stall-regulated wind turbines [24]. The three dimensional stall model employed in WTAC is identical to the one in AirfoilPrep [25] which includes the modifications from Du and Selig [26] and Eggers et al [27]. Moreover, Larsen et al. dynamic stall model is used [28]. A conservative variable speed control strategy $[14,19]$ is chosen in order to limit the potential power peaks. The control strategy varies accordingly to the wind turbine operating region as illustrated in Figure 3: (1) the maximum power coefficient is tracked in low wind speeds, (2) the generator torque increases in order to force the blades into stall and (3) as the wind increases above rated the power is limited by reducing the wind turbine angular speed and forcing the blades into stall. 
Macquart T \& Maheri A, A stall-regulated wind turbine design to reduce fatigue. Published in Renewable Energy 2018

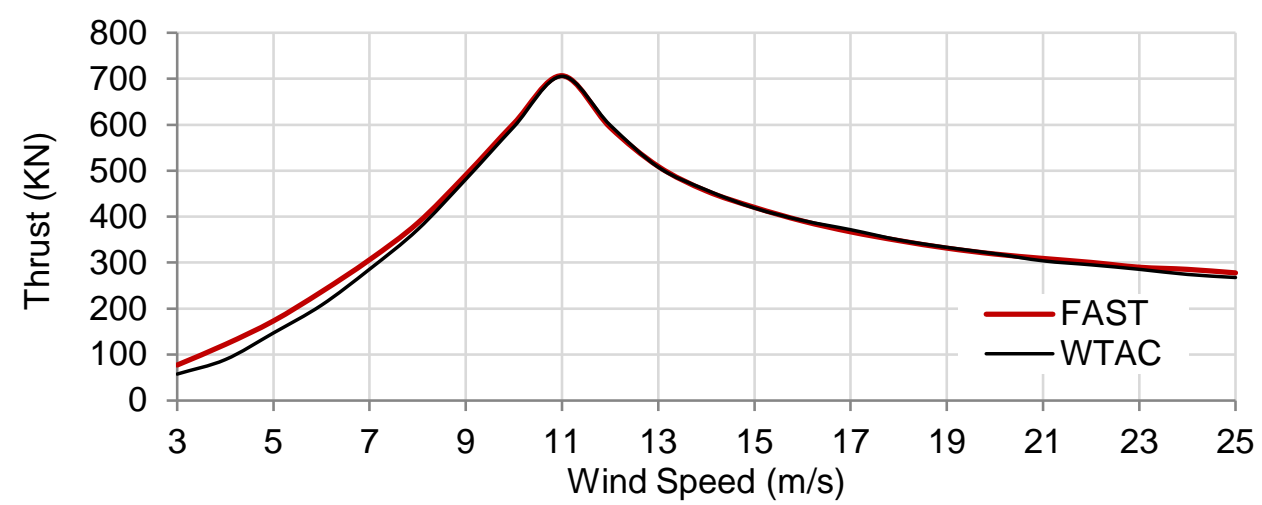

(a)

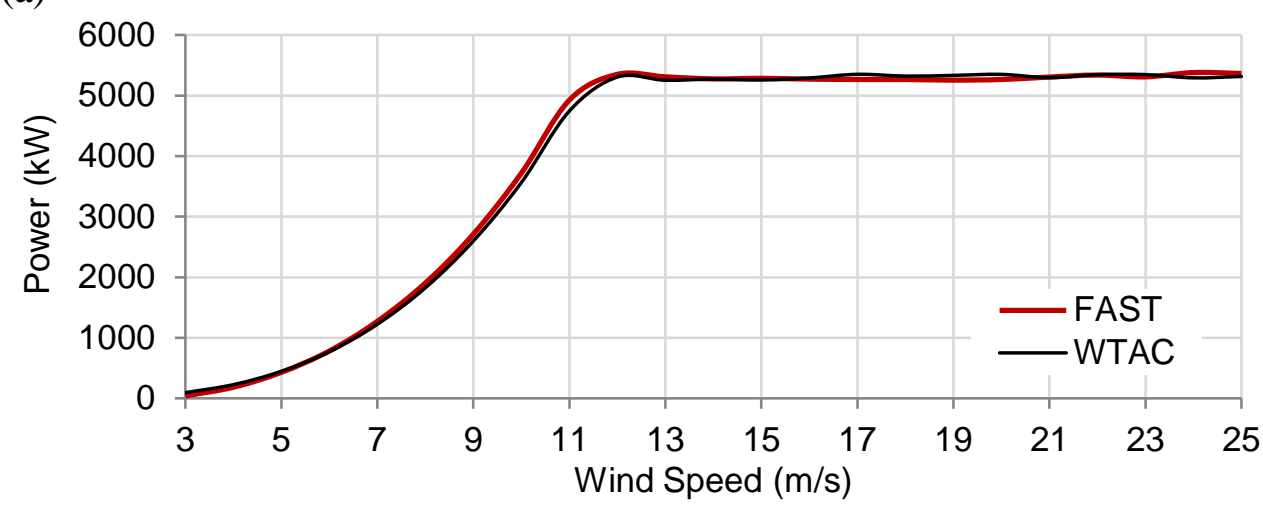

(b)

93

94

95

96

97

Figure 1-Power and thrust curve comparison between WTAC and FAST predictions for the 5MW VS-PC wind turbine

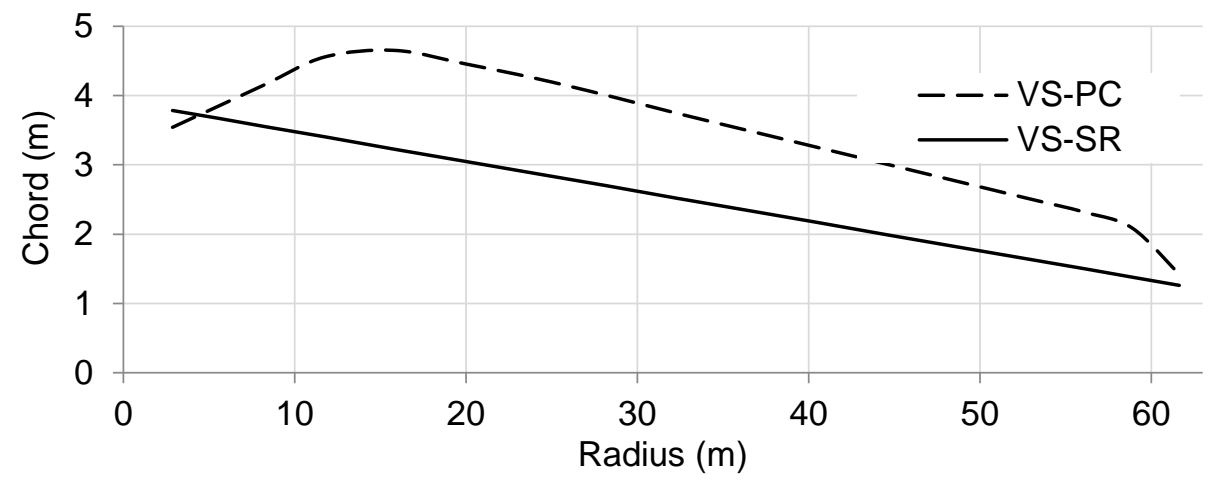

(a)

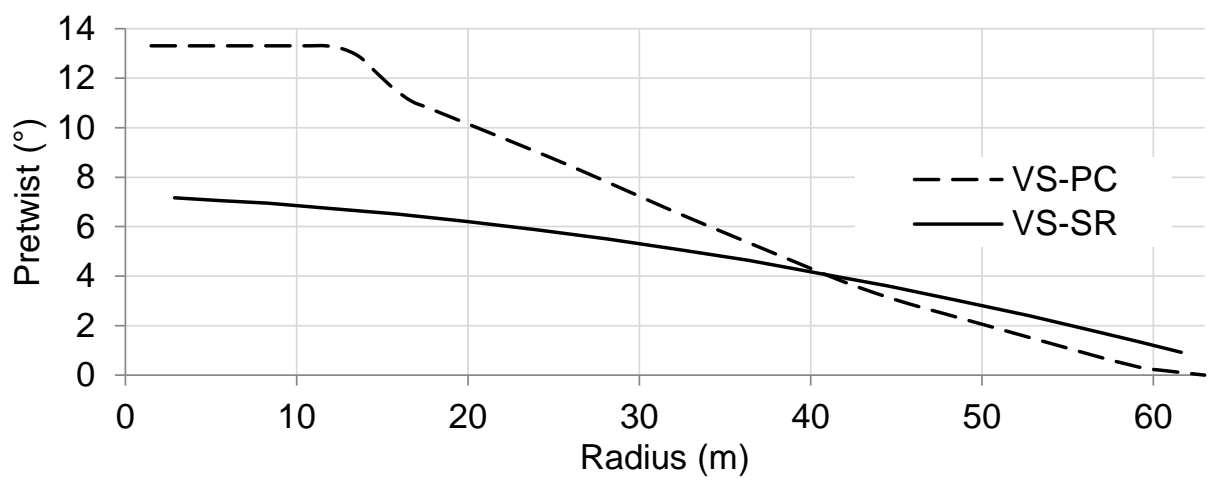

(b) 
Table 1-Aerofoil distribution for the VS-SR and VS-PC 5MW wind turbine designs

\begin{tabular}{c|c|c}
\multirow{2}{*}{ Radius (m) } & \multicolumn{2}{|c}{ Aerofoils } \\
\cline { 2 - 3 } & VS-PC & VS-SR \\
\hline 2.867 & Root Cylinder & Root Cylinder \\
5.600 & Root Cylinder & Root Cylinder \\
8.333 & Root Cylinder & Root Cylinder \\
11.750 & DU40_A17 & DU40_A17 \\
15.850 & DU35_A17 & DU40_A17 \\
19.950 & DU35_A17 & DU40_A17 \\
24.050 & DU30_A17 & DU40_A17 \\
28.150 & DU25_A17 & DU25_A17 \\
32.250 & DU25_A17 & DU25_A17 \\
36.350 & DU21_A17 & DU25_A17 \\
40.450 & DU21_A17 & DU25_A17 \\
44.550 & NACA 64-618 & NACA 64-618 \\
48.650 & NACA 64-618 & NACA 64-618 \\
52.750 & NACA 64-618 & NACA 64-618 \\
56.166 & NACA 64-618 & NACA 64-618 \\
58.900 & NACA 64-618 & NACA 64-618 \\
61.634 & NACA 64-618 & NACA 64-618
\end{tabular}

102

103

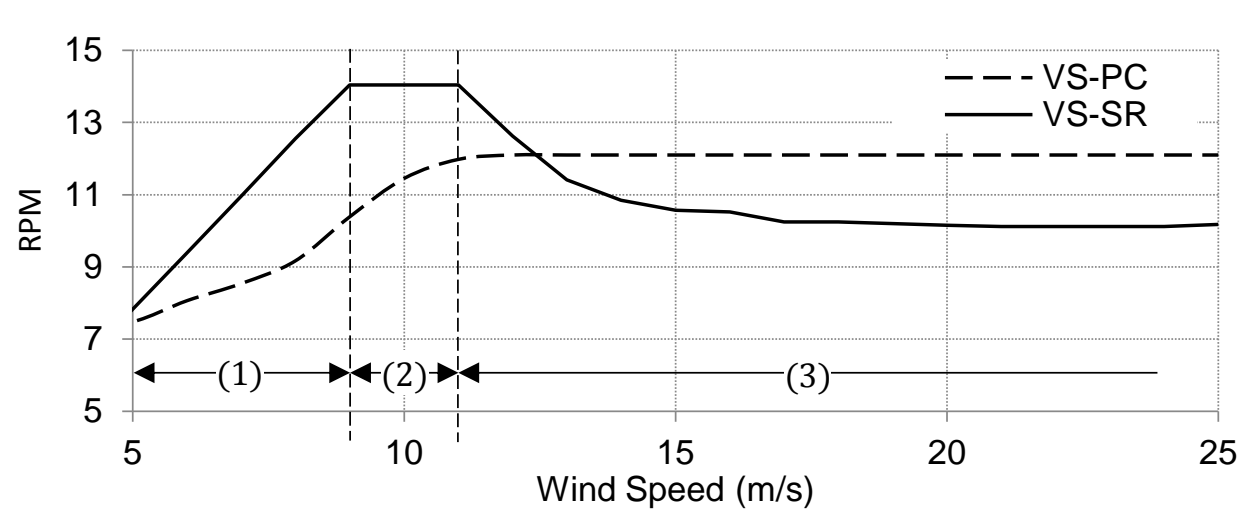

Figure 3-Variable-speed control strategy for the 5MW VS-PC and VS-SR wind turbines

Employing WTAC, a VS-SR wind turbine equipped with the blades of Table 1 while operating according to the variable-speed control strategy of Figure 3 is simulated. The steady state power and thrust are presented in Figure 4.

From the power curve of Figure 4.a, we see that the VS-SR design is highly efficient at generating and limiting power, although small power losses are observed near rated wind speed. Figure 4.b shows that the VS-SR wind turbine experiences a smaller pick thrust load compared to the VS-PC design. This is due to specific blade and control design. In wind speeds below the rated speed, the VS-SR wind turbine operates at higher rotor speeds but the blade operates mainly in lower angles of attack, where the drag force is smaller. Although a higher rotor speed leads to higher dynamic pressure, but since the drag coefficient is very small in low angles of attack, the overall drag force on the blade and consequently the thrust load on the rotor is less than that of the VS-PC turbine. In higher speeds, having the blade operating in higher angles of attack and entering stall region, significant 
increase in the drag coefficient is expected. However, due to operating at lower rotor speeds compared to the VS-PC design, the thrust force does not increase sharply.

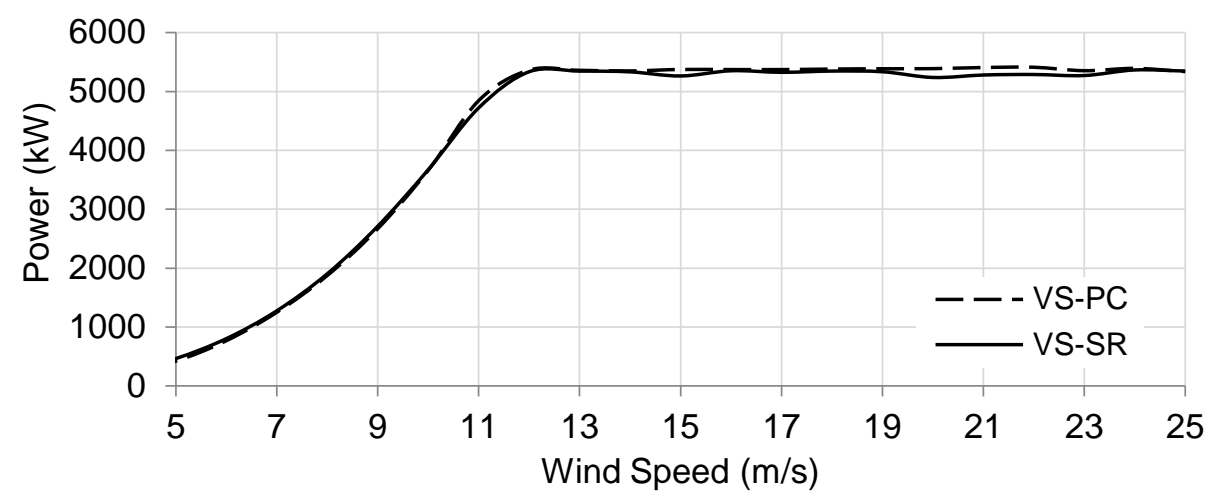

(a)

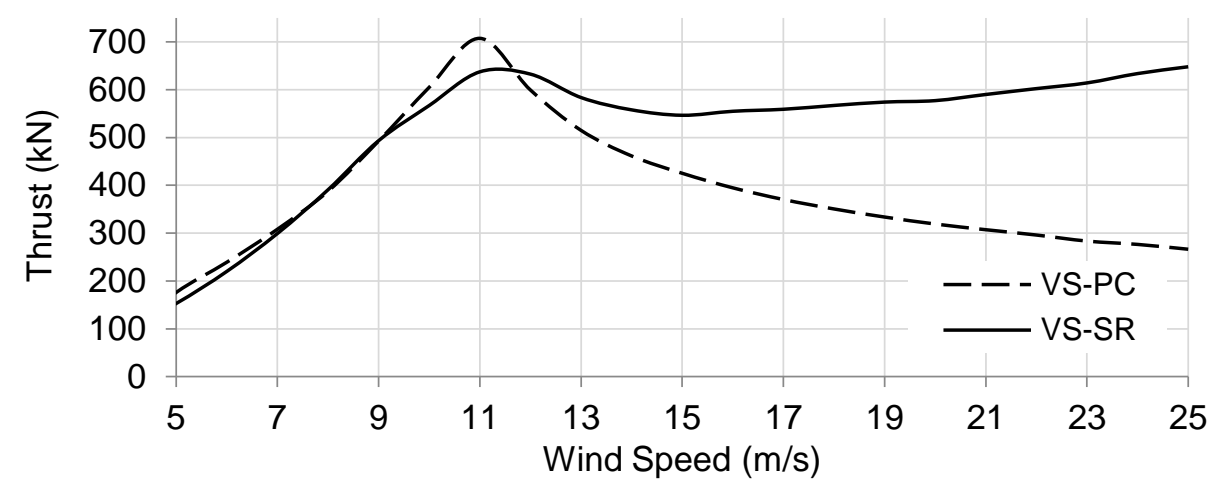

(b)

Figure 4-Comparison between the VS-PC and VS-SR power and thrust curves

\section{Aerodynamic Sensitivity and Fatigue}

The aerodynamic sensitivity of a wind turbine operating at a particular mean wind speed is obtained by changing the incoming wind speed while keeping all other parameters constant. In other words, the aerodynamic sensitivity reflects the variation of the wind turbine power when subjected to an instantaneous change in velocity and aerodynamic torque. Results for mean wind speeds of 5, 10, 15, 20 and $25 \mathrm{~m} / \mathrm{s}$ are presented in Figure 5. Moreover, the approximated slope of each curve is given in Table 2.

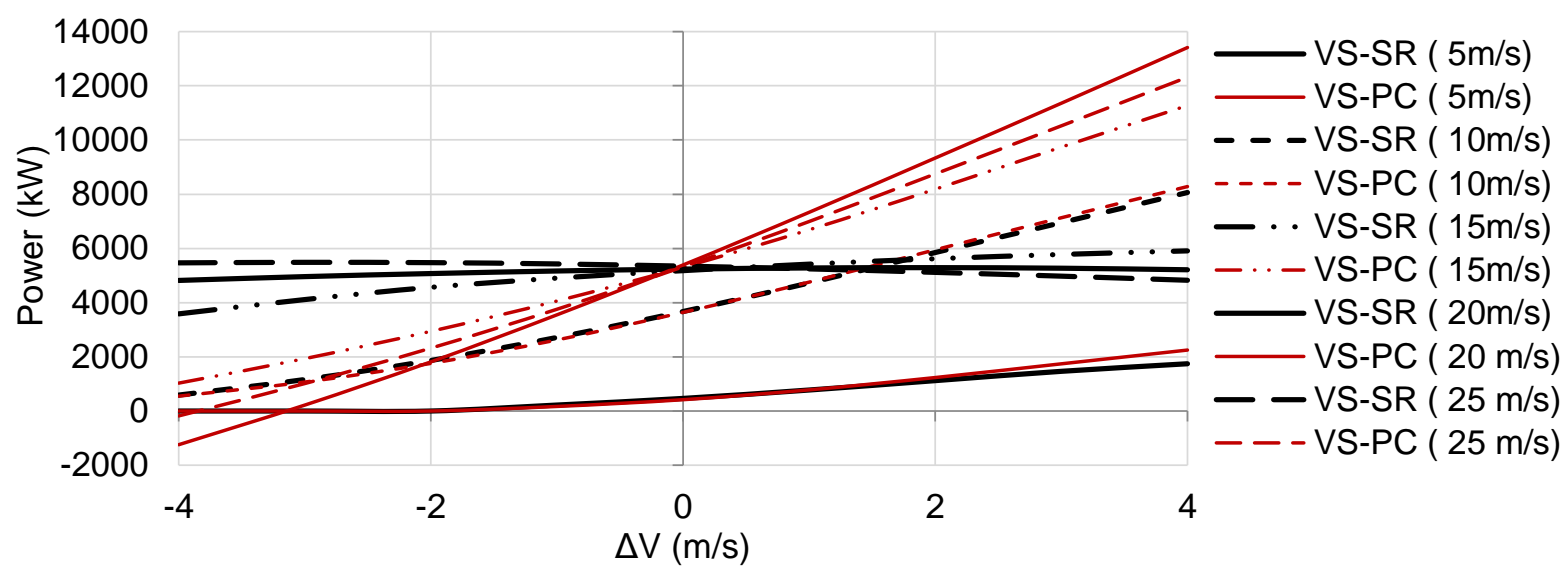

Figure 5- Comparison between the aerodynamic sensitivity of the VS-PC and the VS-SR wind turbines 
Table 2-Aerodynamic sensitivity of the VS-PC and the VS-SR wind turbines

\begin{tabular}{c|c|c}
\multirow{2}{*}{$\begin{array}{c}\text { Wind Speed } \\
(\mathrm{m} / \mathrm{s})\end{array}$} & \multicolumn{2}{|c}{ Slope: $d p / d(\Delta V)\left(\mathrm{kW} / \mathrm{ms}^{-1}\right)$} \\
\cline { 2 - 3 } & VS-PC & VS-SR \\
\hline 5 & 288 & 236 \\
10 & 994 & 954 \\
15 & 1292 & 282 \\
20 & 1577 & 51 \\
25 & 1847 & -83
\end{tabular}

As it can be observed in Figure 5, the aerodynamic sensitivities of the two designs are similar for low and medium wind speeds. That is, in low wind speeds both designs maximise the power coefficient and aerodynamic performance therefore resulting in high aerodynamic sensitivity. On the other hand, for higher wind speeds the aerodynamic sensitivity of the VS-PC turbine keeps increasing whereas the sensitivity of the VS-SR turbine decreases. The blades aerodynamic insensitivity comes from the insensitivity of its aerofoils to change in flow conditions. For instance,

Figure 6 shows that as the aerofoil NACA 64-618 enters into stall, the slope of the lift coefficient approaches zero. Blades on pitch-controlled systems mostly operate in pre-stall region where the lift slope is maximal whereas the blades of a stall-regulated wind turbine operate in both pre-stall and stall. This is clearly illustrated in Figure 7 that shows the angle of attack distribution along the blade span for the various operating mean wind speeds of the VS-SR wind turbine. The progressive stall line indicates both the stall location along the blade span and the steady state wind speed at which it occurs. Typically, when the blades of a pitch-controlled wind turbine are subjected to a sudden change in wind speeds the angle of attack variation results in a large change in lift and its corresponding aerodynamic forces. On the other hand, when a blade operating along or above the progressive stall line is subjected to the same event, it experiences a greater change in drag and a lower change in lift (see Figure 6). The VS-SR wind turbine blades aerodynamic sensitivity decreases as the angle of attack increases and the blades progressively enters into stall. The resulting change in aerodynamic forces experienced by the stalled blades is therefore lower than the one experienced by the pitchcontrolled wind turbine.

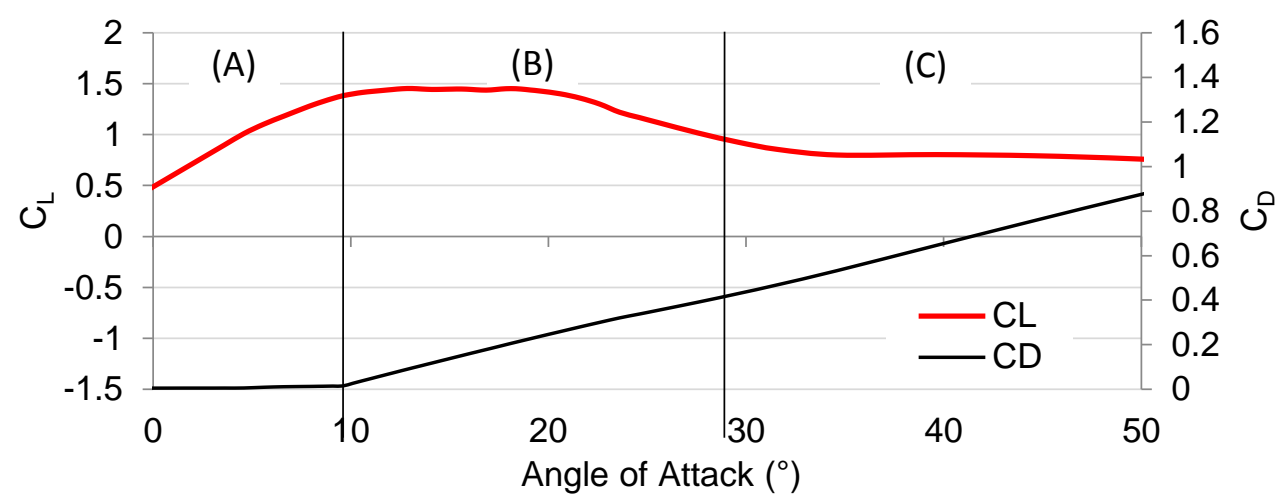

Figure 6-Lift and drag coefficient of the aerofoil NACA 64-618

FAST is used to calculate the flapwise root bending moments experienced by the VS-PC and VS-SR wind turbines operating in windshear conditions. In this preliminary study, the structural properties of both blades are assumed identical. Results are presented in Figures 8 and 9. Figure 8 compares the root bending moment experienced by the blades at two wind speeds of 12 and $21 \mathrm{~m} / \mathrm{s}$. Figure 9 compares the magnitude of the cyclic (alternating) component of the loads on the blades of the two 
175

176

177

178

179

180
181

182

183

184

types of wind turbines in the range of 5 to $25 \mathrm{~m} / \mathrm{s}$. As these figures show, the aerodynamic sensitivity and the cyclic loads amplitude of the stall-regulated wind turbine decreases with wind speeds (see Figures 8.b and 9). On the other hand, as the aerodynamic sensitivity of the pitch-controlled wind turbine increases with wind speeds the cyclic loads amplitude also increases as shown in Figure 9.

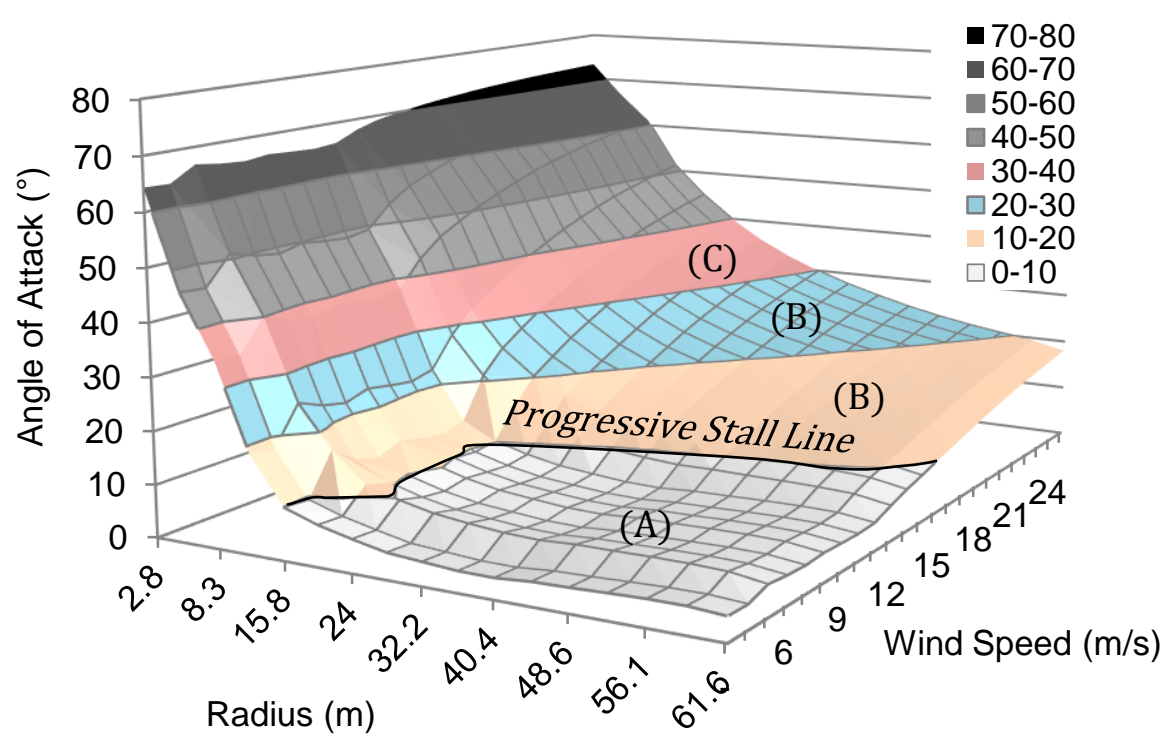

Figure 7-Angle of attack distribution along the blade span and over the entire operating mean wind speed range for the VS-SR wind turbine

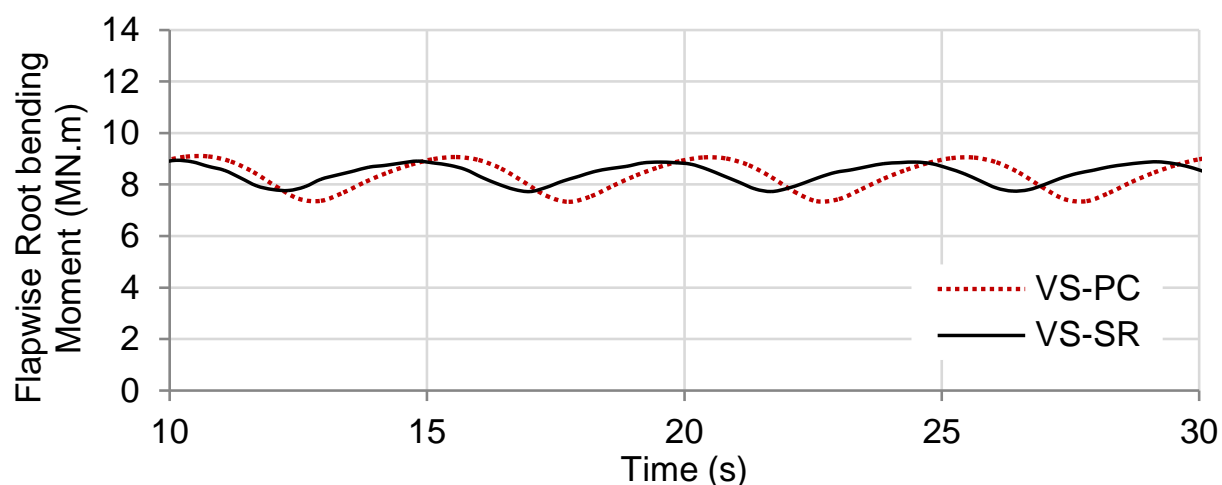

(a)

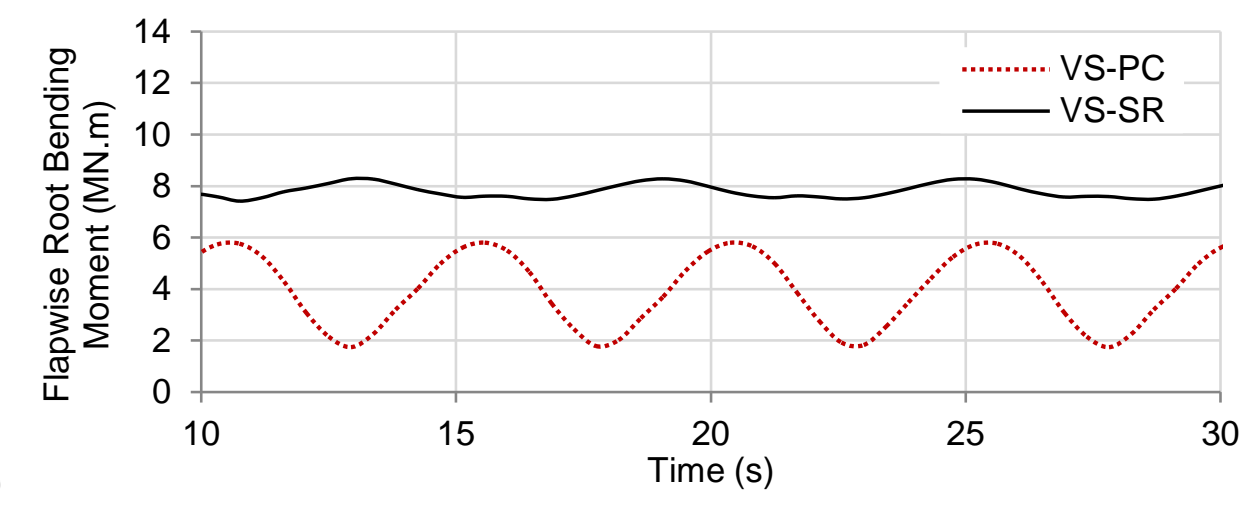

(b)

Figure 8-Comparison of the flapwise root bending moments for the two wind turbine designs at wind speeds of (a) 12 and (b) $21 \mathrm{~m} / \mathrm{s}$ 


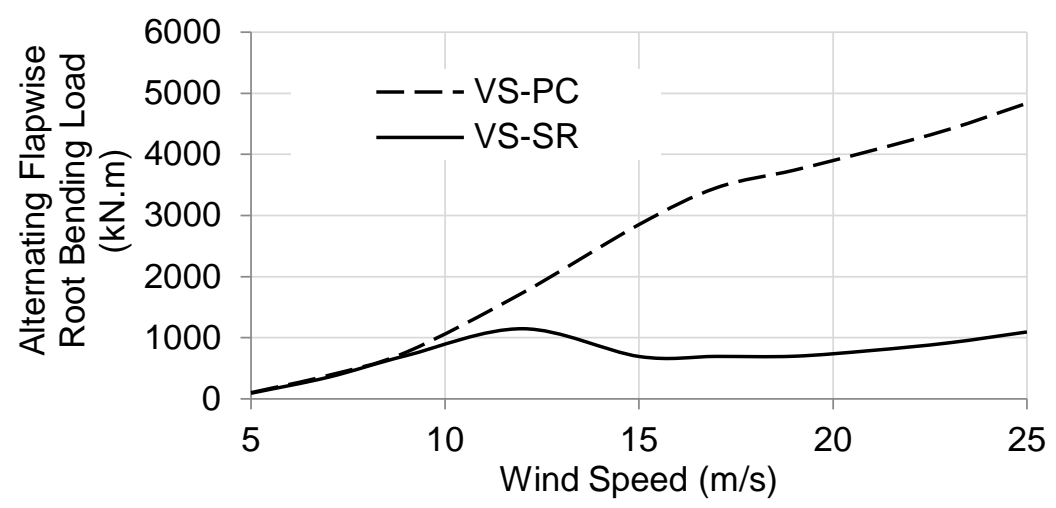

Figure 9-Comparison between the magnitudes of the cyclic (alternating) component of the root bending moment of the two wind turbines

For a given blade, the fatigue life depends on cyclic and stochastic behaviour of loads, namely the number of fluctuations (low frequency cyclic and high frequency turbulence), the alternating force components, and of the second degree of importance compared to the alternating components, the midrange force component. With reference to Figures 3 and 9, one can see that at lower wind speeds (less than $9 \mathrm{~m} / \mathrm{s}$ ), the proposed VS-SR design experiences higher rotational speeds- which directly translates to a higher number of cycles- and is subjected to more or less the same alternating force components. Taking into account only this region and excluding the effect of high frequency fluctuations, one can argue that the proposed VS-SR blade design performs inferior to its VS-PC counterpart. However, this argument weakens by considering the inherent insensitivity of stallregulated blades to fluctuating forces due to the turbulence. Moreover, at higher wind speeds above the rated speed, where the loads are generally higher and more damaging, similar argument can be made in favour of VS-SR: lower rotational speed (Figure 3), significantly lower alternating component of cyclic force (Figure 9), and due to insensitivity of the stall-regulated design, significantly lower alternating fluctuating forces due to turbulence (Figure 5). The next section expands on the insensitivity of SR design to high frequency fluctuations by detailing the variable speed control strategy and power/torque transients.

\section{Power Transient and Variable-Speed Control Strategy}

Compared to VS-PC wind turbines where the pitch angle can rapidly be controlled to shed power, avoiding peak power is more critical for VS-SR turbines. Consequently the transitions between the different control regions need to be carefully designed in order to ensure safe operating conditions. The dynamic behaviour of VS-SR turbines for low wind speeds has been previously demonstrated in literature [4] and is not reported herein.

The original set-up for the variable speed operation of the NREL 5MW wind turbine is modified for the VS-SR design. Due to a lower rated angular speed compared to the VS-PC design, the rated generator torque is increased by $16 \%$ to obtain a rated value of $50 \mathrm{kN}$.m. The maximum allowable torque is fixed at $10 \%$ above rated $(55 \mathrm{kN} . \mathrm{m})$. The low shaft rated angular speed is fixed at $10.1 \mathrm{rpm}$ (i.e. high wind speed $\mathrm{rpm}$ ) with a variable speed operation range of $\pm 40 \%[14,15]$. The original drive train ratio (97:1), generator efficiency $(94.4 \%)$, rotor inertia and other parameters are kept identical. The turbulent wind fields used for the dynamic simulations presented in the rest of this paper have been generated using TurbSim [29].

The wind turbine control near rated wind speeds is divided into four transitions as illustrated in Figure 10. The transitions $\left(t_{1}\right)$ and $\left(t_{2}\right)$ correspond to a change between the low and medium wind speed regions. There are no major challenges for these transitions because the generator torque control margin is sufficient in order to follow the desired control strategy: $\left(\mathrm{t}_{1}\right)$ the power increases towards 
rated while the rpm also increases or $\left(\mathrm{t}_{2}\right)$ the aerodynamic torque and power decreases away from rated values. The transition $\left(\mathrm{t}_{3}\right)$ refers to the transition from the medium to the high wind speed region when the wind speed increases. This is the most critical transition because the aerodynamic torque increases whereas the rpm must decrease to limit power. As a consequence, there is a risk that a sudden increase in wind speed and therefore aerodynamic torque results in a large peak power above rated. It is crucial to avoid such scenario that could seriously damage the wind turbine. The variable speed control strategy must be designed considering the trade-off between the desired rapid changes in angular speed and the sudden power increase. The transition $\left(\mathrm{t}_{4}\right)$ corresponds to a variation of wind speeds from the high to the medium wind speeds. Caution must also be taken during this transition because the aerodynamic torque has reached rated value and the generator torque control margin is therefore small. Rapid increase of the rpm should be avoided to limit peak power when a quick $\left(\mathrm{t}_{4}\right)$ transition is followed by a $\left(\mathrm{t}_{3}\right)$ transition.

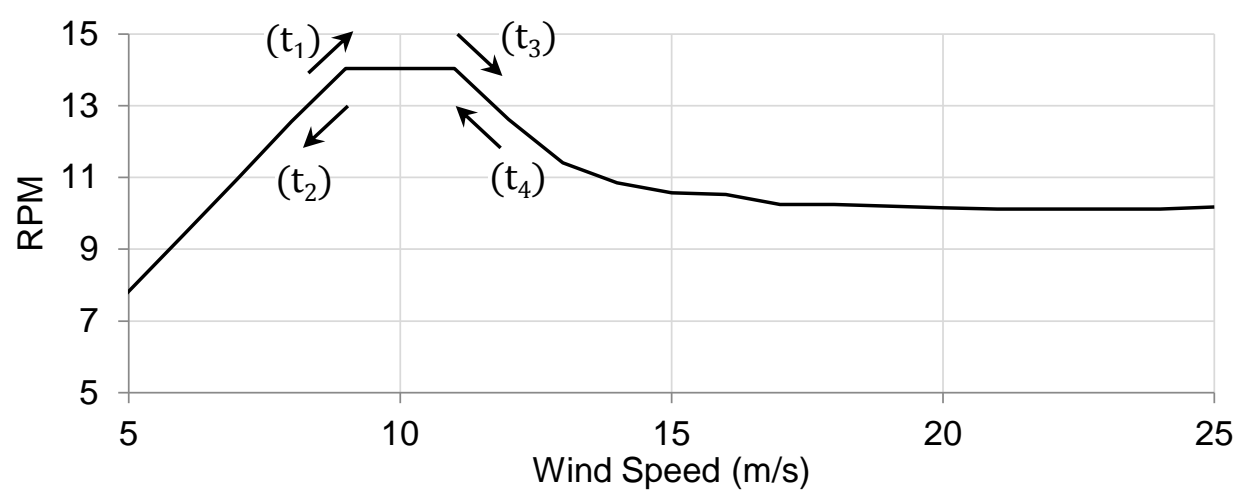

Figure 10-Variable-speed control transitions for the stall regulated wind turbine

Applying those limitations to the variable speed control strategy proposed by Pierce and Migliore [14], the power transients near and above rated wind speeds are evaluated. Since the main concern is to limit power peaks, the converter and power smoothing control strategy were not modelled. The results of the stall-regulated wind turbine operating near rated wind speeds are presented in Figure 11. As can be observed in Figure 11.a, the wind turbine operates in the critical transition regions $\left(\mathrm{t}_{3}\right)$ and $\left(\mathrm{t}_{4}\right)$ with fast changing wind speeds. Notice that, despite the substantial aerodynamic torque peaks (Figure 11.c, time $\approx 50-70$ s), the power generator is well-limited to $+10 \%$ rated power as illustrated in Figure 11.b.

The control strategy trade-off between power limitation and generation may result in power losses due to the limitation at which the rpm is allowed to change as it can be seen in Figures 11.a and 11.b. For instance, between 50 and 80 seconds the aerodynamic torque rapidly decreases before substantially increasing for 20 seconds. First the generator torque is controlled such that the rpm increases to bring the power back to rated value $(\approx 65 \mathrm{~s})$. At that point, the aerodynamic torque keeps increasing while the generator torque is controlled to reduce the rpm and maintain power at rated. However, the rate at which the rpm decreases is limited by the rotor inertia and a small power overshoot occurs. Note that if the rpm was allowed to quickly increase during the first aerodynamic torque drop, the rpm would have reached a higher value and the power overshoot would have been more significant. That is, the acceleration and deceleration rates of the angular speed during the $\left(\mathrm{t}_{3}\right)$ transition are critical to avoid power peaks. Due to this conservative control strategy the rotor angular speed near rated wind speed is often lower than predicted by the steady state design (Figure 11.a). As a consequence, a lower generator variable speed operation range could be used. Although this would results in much lower power generation when using a steady state analysis, the stored rotor kinetic energy helps in maintaining power near rated in a dynamic framework. 


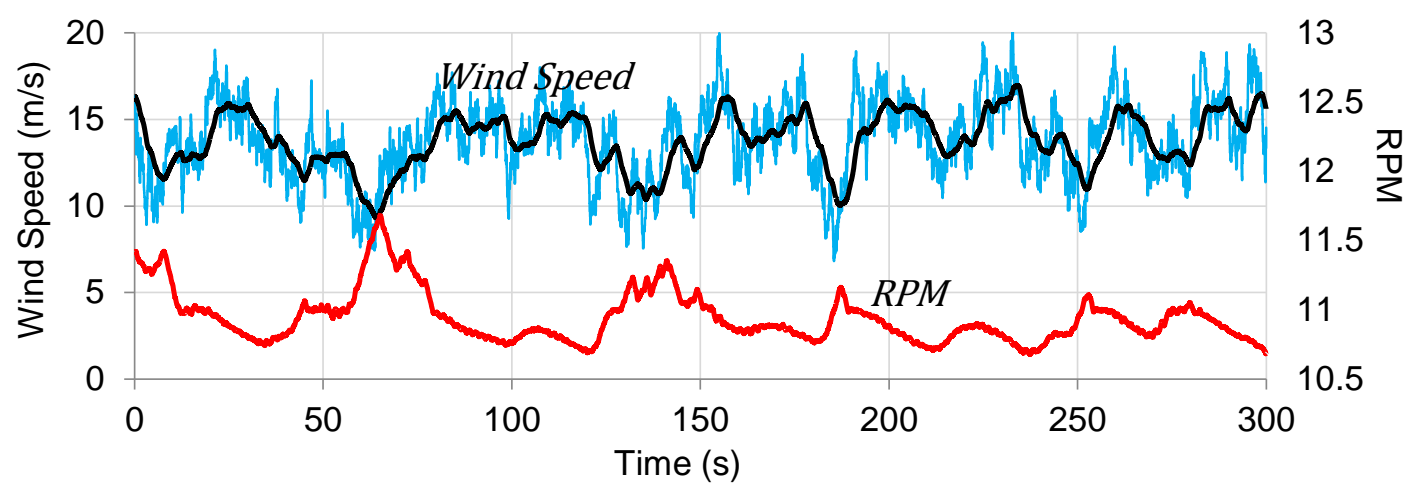

(a)

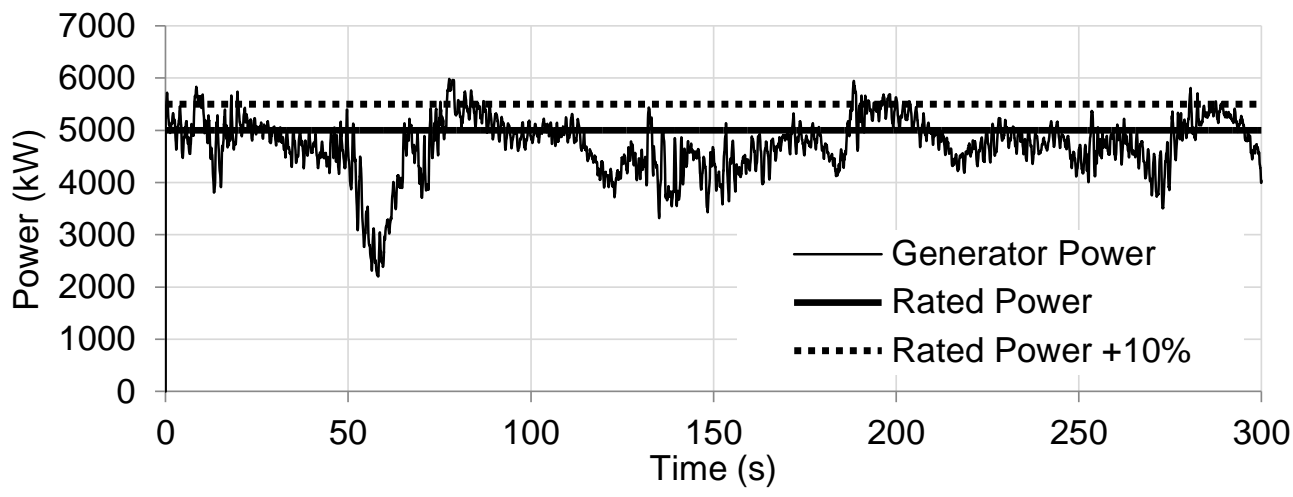

(b)

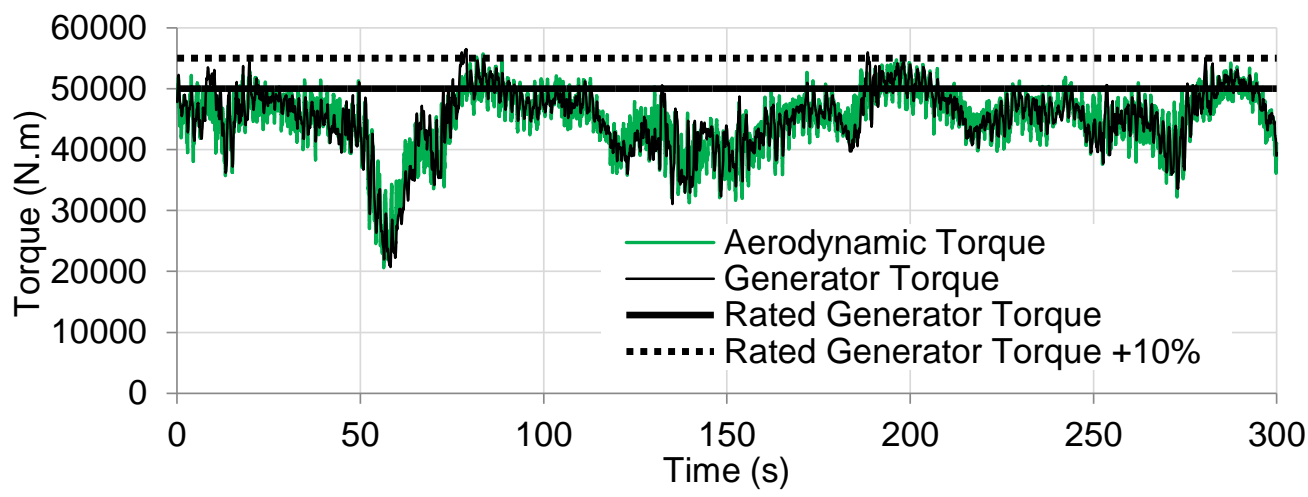

(c)

Figure 11-Dynamic performance of the VS-SR wind turbine operating near rated wind speed, (a) wind speed at hub and rpm, (b) power and (c) torque

Results for the stall-regulated wind turbine operating well above rated wind speed are presented in Figure 12. As shown in Figure 12.a the wind turbine is subjected to a rapidly varying wind fields in the high wind speed region. As expected from the aerodynamic sensitivity results presented in Section 3 , the wind turbine power and aerodynamic torque are not very sensitive to change in wind speeds above $15 \mathrm{~m} / \mathrm{s}$ (see Figures 12.b and 12.c). Consequently, the torque and power transients in high wind speeds are minimal. Furthermore, one can observe that the power is well-maintained below $+10 \%$ of its rated value. 


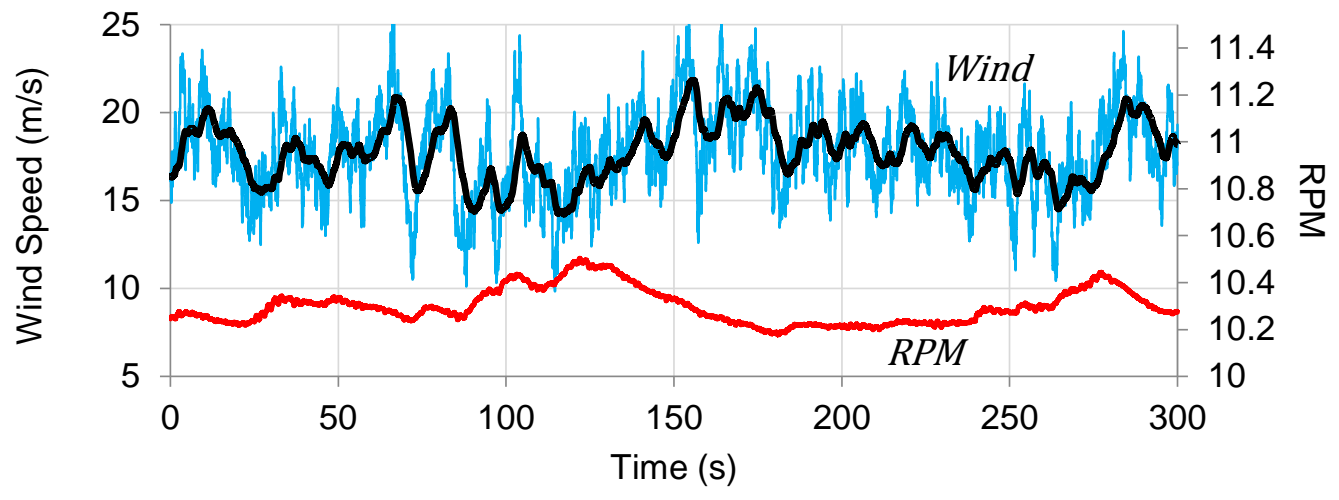

(a)

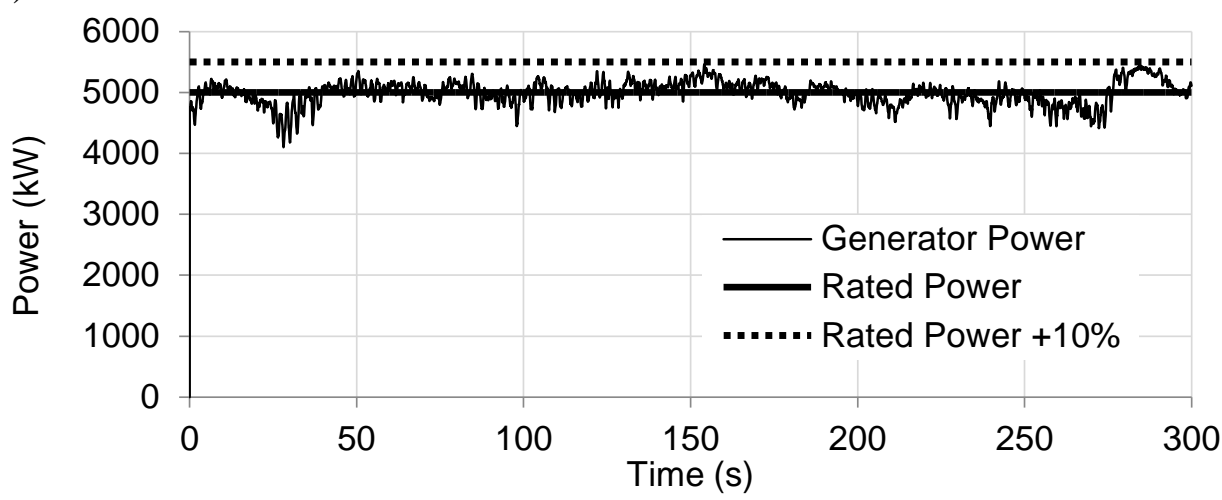

(b)

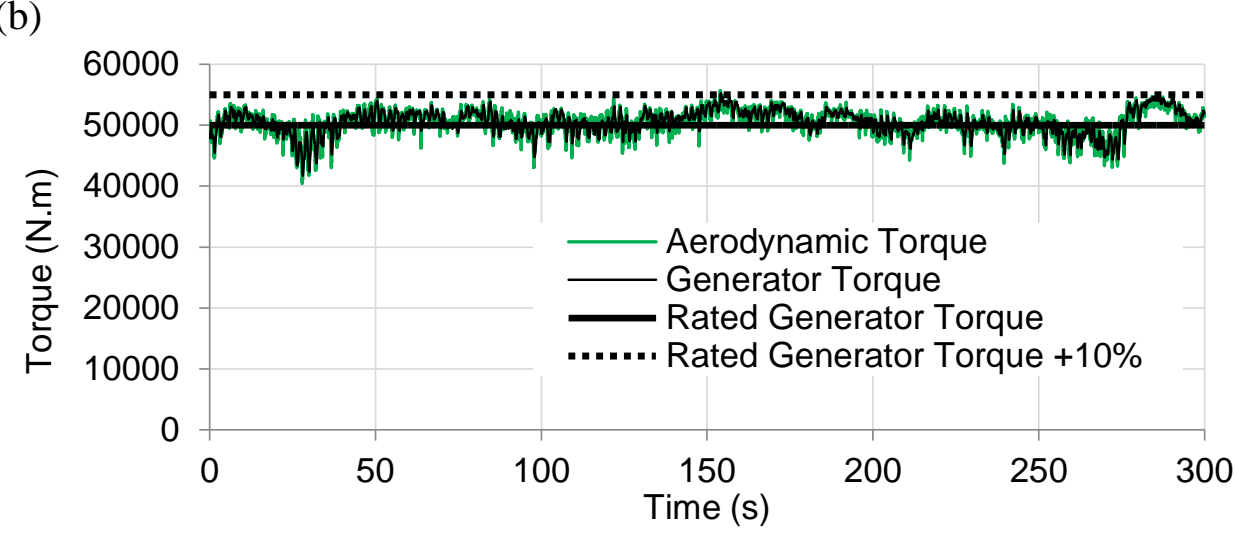

(c)

Figure 12-Dynamic performance of the VS-SR wind turbine operating in high wind speed, (a) wind speed at hub and rpm, (b) power and (c) torque

\section{Concluding Remarks}

The present paper investigates the potential benefit of variable-speed stall-regulated wind turbines in reducing fatigue loads and limiting power transients. While the potential interest in using stallregulated wind turbines for that purpose was speculated, no thorough investigation had been previously carried out. During this investigation, it was found that VS-SR wind turbine can be as efficient as a pitch-control design in power generation and regulation, and that VS-SR turbines can be designed to experience the same (or even slightly lower) thrust load compared to a pitch-control design. Most importantly, it was shown that by taking advantage of the aerodynamic insensitivity of stalled blades, VS-SR designs experience significantly lower fatigue loads than pitch-controlled wind turbines. Furthermore, it was shown that the VS-SR design reported in this paper helps in minimising the power transients near and above rated wind speed. The power generated was shown to be wellmaintained around its rated value while operating under highly turbulent winds. 
Compared to pitch controlled turbines, stall-regulated turbines generally experience higher blade loading at higher wind speeds and produce more noise. Higher blade loading leads to heavier blades, which is a major drawback of stall-regulated turbines. The VS-SR design proposed in this investigation is the results of a steady state aerodynamic optimisation problem without including structural design parameters. The structure of the VS-SR turbine was assumed identical to the VSPC blade. It is, therefore, most likely that a comprehensive optimisation-including structural optimisation- will result in a VS-SR design with larger chord and/or thicker shell, and/or a thicker aerofoil family. Furthermore, the behaviour of VS-SR wind turbines is strongly dictated by unsteady aerodynamics. Consequently, unsteady-based design optimisations may be required in order to achieve optimal performance under unsteady conditions. Moreover, the progressive stall line may be used as a design variable in order to obtain the desired wind turbine aerodynamic sensitivity. A natural extension of the presented work is therefore an integrated design approach, in which the optimisation problem is formulated to include both steady and unsteady aerodynamic performance measures and structural performance measures simultaneously.

\section{References}

1. Barlas TK, van Kuik G. Review of state of the art in smart rotor control research for wind turbines. Progress in Aerospace Sciences 2010, 46(1): p. 1-27. DOI: 10.1016/j.paerosci.2009.08.002

2. Barlas TK, van Kuik G. State of the art and prospectives of smart rotor control for wind turbines. Journal of Physics: Conference Series 2007. IOP Publishing. DOI:10.1088/1742$6596 / 75 / 1 / 012080$

3. Larsen TJ, Madsen HA, Thomsen K. Active load reduction using individual pitch, based on local blade flow measurements. Wind Energy 2005. 8(1): p. 67-80. DOI: 10.1002/we.141

4. Muljadi E, Butterfield CP. Pitch-controlled variable-speed wind turbine generation. Industry Applications, IEEE Transactions 2001. 37(1): p. 240-246. DOI: 10.1109/28.903156

5. Castaignet D, Couchman I, Poulsen NK, Buhl T, Wedel-Heinen JJ. Frequency-Weighted Model Predictive Control of Trailing Edge Flaps on a Wind Turbine Blade. IEEE transaction on control system technology 2013. 21(4). DOI: 10.1109/ACC.2011.5991111

6. Kragh KA, Hansen MH. Load alleviation of wind turbines by yaw misalignment. Wind energy, 2013.

7. Johnson SJ, Baker JP, van Dam CP, Berg D. An overview of active load control techniques for wind turbines with an emphasis on microtabs. Wind Energy 2010. 13(2-3): p. 239-253. DOI: $10.1002 /$ we.356

8. Macquart T, Maheri A, Busawon K. Microtab dynamic modelling for wind turbine blade load rejection. Renewable Energy 2014. 64: p. 144-152. DOI: 10.1016/j.renene.2013.11.011

9. Maheri A, Isikveren AT. Performance prediction of wind turbines utilizing passive smart blades: approaches and evaluation. Wind energy 2010. 13(2-3): p. 255-265. DOI: $10.1002 /$ we. 340

10. Castaignet D, Barlas T, Buhl T, Poulsen NK, Wedel-Heinen JJ, Olesen NA, Bak C, Kim T. Full-scale test of trailing edge flaps on a Vestas V27 wind turbine: active load reduction and system identification. Wind energy 2014. 17(4): p. 549-564. DOI: 10.1002/we.1589

11. Muljadi E, Butterfield CP, Buhl ML. Effects of turbulence on power generation for variablespeed wind turbines 1996: National Renewable Energy Laboratory.

12. Muljadi E, Pierce KG, Migliore P. Control strategy for variable-speed, stall-regulated wind turbines. American Control Conference 1998. Proceedings of the 1998. 1998. IEEE.

13. Muljadi E, Pierce KG, Migliore P. A conservative control strategy for variable-speed stallregulated wind turbines 2000, National Renewable Energy Laboratory.

14. Pierce KG, Migliore P. Maximizing energy capture of fixed-pitch variable-speed wind turbines2000, National Renewable Energy Laboratory. 
15. Carlin PW, Laxson AS, Muljadi E. The history and state of the art of variable-speed wind turbine technology. Wind energy 2003. 6(2): p. 129-159. DOI: 10.1002/we.77

16. Bang D, Polinder H, Ferreira JA, van Rooij R. New active speed stall control compared to pitch control for a direct-drive wind turbine. Europe, 2007. 5: p. 11.

17. Merz KO. Conceptual design of a stall-regulated rotor for a deepwater offshore wind turbine. Doctoral Thesis. NTNU, 2011.

18. Giguère P, Tangler J, Selig M. Blade design trade-offs using low-lift airfoils for stall-regulated HAWTs. Journal of solar energy engineering, 1999. 121(4): p. 217-223. DOI:10.1115/1.2888170

19. Bulder BH, Hendriks HB, van Langen PJ, Lindenburg C, Snel H, Bauer P, Polinder H, van Rooij R, Subroto H, Zaayer MB. The ICORASS feasibility study. ECN Wind energy 2013. 2012: p. 2011.

20. Macquart T, Maheri A. Integrated aeroelastic and control analysis of wind turbine blades equipped with microtabs, Renewable Energy 75 (2015) 102-114

21. Jonkman J, Buhl M. FAST user's guide. Golden, CO: National Renewable Energy Laboratory (2005).

22. Jonkman J, Butterfield S, Musial W, Scott G. Definition of a 5-MW reference wind turbine for offshore system development 2009, National Renewable Energy Laboratory Colorado.

23. Snel H, Houwink R, Bosschers J. Sectional prediction of lift coefficients on rotating wind turbine blades in stall1994, Netherlands Energy Research Foundation.

24. Lindenburg C. Modelling of rotational augmentation based on engineering considerations and measurements. European Wind Energy Conference, London. 2004.

25. Hansen C. NWTC design codes (AirfoilPrep), 2012.

26. Du Z, Selig MS. A 3-D stall-delay model for horizontal axis wind turbine performance prediction. Proceedings of the 1998 ASME wind energy symposium, Reno, NV (pp. 9-19).

27. Eggers A, Chaney K, Digumarthi R. An assessment of approximate modeling of aerodynamic loads on the UAE rotor. ASME 2003 Wind Energy Symposium. 2003. American Society of Mechanical Engineers. DOI:10.1115/WIND2003-868

28. Larsen JW, Nielsen SRK., Krenk S. Dynamic stall model for wind turbine airfoils. Journal of Fluids and Structures 2007. 23(7): p. 959-982. DOI: 10.1016/j.jfluidstructs.2007.02.005

29. Foley JT, Gutowski TG. TurbSim: Reliability-based wind turbine simulator. 2008 IEEE International Symposium on Electronics and the Environment 2008: p. 315-319. DOI: 10.1109/ISEE.2008.4562872 